\title{
MicroRNA-524-5p suppresses the growth and invasive abilities of gastric cancer cells
}

\author{
GUANG-HUI LIU* , YUAN-HUA LIU* , ZHEN YANG, A-LI ZHU and CHUN-LIN ZHAO \\ Department of Gastrointestinal Surgery, The First Affiliated Hospital of Zhengzhou University, \\ Zhengzhou, Henan 450052, P.R. China
}

Received November 26, 2014; Accepted November 30, 2015

DOI: $10.3892 / \mathrm{ol} .2016 .4143$

\begin{abstract}
Previous studies have demonstrated that microRNAs (miRNAs) are associated with tumor development and progression. miRNA-524-5p (miR-524-5p) has been reported to be involved in the development and progression of several types of cancer, but its role in gastric cancer has not been fully elucidated to date. Therefore, the aim of the present study was to investigate the expression levels and function of miR-524-5p in human gastric cancer. The expression levels of miR-524-5p were assessed in gastric cancer specimens and cell lines, including MKN-45, SGC-7901 and MGC-803 cell lines and gastric epithelial mucosa GES-1 cells, using reverse transcription-quantitative polymerase chain reaction (RT-qPCR). Cell proliferation and cell apoptosis assays and invasion analysis in gastric cancer cell lines were performed to evaluate the effects of miR-524-5p on gastric cancer cells in vitro. The expression levels of matrix metallopeptidase (MMP)-2 and MMP-9 were determined by RT-qPCR and western blot analysis. The expression of miR-524-5p was significantly decreased in gastric cancer tissues and cell lines. Additionally, the results of the in vitro experiments demonstrated that overexpression of miR-524-5p inhibited cell proliferation and invasion, and promoted cell apoptosis in gastric cancer cells. Human gastric cancer SGC-7901 and MGC-803 cell lines transfected with miR-524-5p exhibited reduced expression levels of MMP-2 and MMP-9. Taken together, the results of the present study indicated that miR-524-5p may function as a novel tumor suppressor gene in gastric cancer, and may serve as a biomarker and therapeutic target for the treatment of gastric cancer.
\end{abstract}

Correspondence to: Dr Chun-Lin Zhao, Department of Gastrointestinal Surgery, The First Affiliated Hospital of Zhengzhou University, 1 Jianshe East Road, Zhengzhou, Henan 450052, P.R China

E-mail: chunlinzhao@126.com

*Contributed equally

Key words: miR-524-5p, gastric cancer, proliferation, apoptosis, invasion

\section{Introduction}

Gastric cancer is one of the most common types of cancer, and the second leading cause of cancer-associated mortality worldwide (1). Almost half of the patients with gastric cancer are Chinese, the majority of whom are diagnosed when the disease has progressed to advanced stages, due to the non-specific symptoms, including epigastric pain, anorexia and vomiting, that present during the early stages of the disease (2). As a result, the overall 5-year survival rate of gastric cancer is $\sim 20 \%$ (3). Numerous studies have investigated the etiology of gastric cancer (4-6). However, the molecular mechanisms that underlie the pathogenesis and progression of gastric cancer remain undefined. Therefore, additional studies are required to elucidate the molecular mechanisms that lead to the metastasis and progression of gastric cancer, and to identify novel markers for the diagnosis, prognosis and treatment of patients with gastric cancer.

MicroRNAs (miRNAs) are short non-coding RNAs of 19-24 nucleotides in length that control the translation and stability of their target messenger (m) RNA by binding to regulatory sites located in the 3'-untranslated region of the transcripts (7). It is widely accepted that miRNAs play pivotal roles in various biological processes, including development, metabolism, cell proliferation, differentiation and apoptosis (8). Numerous studies suggest that there is an association between altered miRNA expression and cancer, since aberrant expression of miRNAs appears to be involved in several important processes that occur during carcinogenesis. Previous studies have investigated the role of miRNA-524-5p (miR-524-5p) in various types of cancer. Chen et al (9) demonstrated that miR-524-5p was associated with overall survival rate and pathological grade of patients with glioma, and Liu et al (10) revealed that the expression of miR-524-5p was reduced in human melanoma, while overexpression of miR-524-5p effectively inhibited melanoma cell proliferation and migration. Furthermore, Liu et al (10) demonstrated that tumors overexpressing miR-524-5p were significantly smaller than those displayed by negative control mice. However, the role of miR-524-5p in gastric cancer remains unclear.

The present study investigated the expression levels of miR-524-5p in human gastric cancer tissues and cell lines, including MKN-45, SGC-7901 and MGC-803 cell lines and gastric epithelial mucosa GES-1 cells. In addition, cell 
proliferation and migration assays, as well as a cell apoptosis analysis, were performed using human gastric cancer SGC-7901 and MGC-803 cell lines to explore the in vitro effects of miR-524-5p in gastric cancer cells.

\section{Materials and methods}

Tissue samples. A total of 15 gastric cancer and adjacent non-cancerous tissue samples were obtained from patients that had undergone surgical treatment for gastric cancer at The First Affiliated Hospital of Zhengzhou University (Zhengzhou, China) between March 2011 and March 2013. The patients were diagnosed independently by two experienced pathologists from The First Affiliated Hospital of Xinxiang Medical University, according to the Cancer Staging Manual published by the American Joint Committee on Cancer. The present study was approved by the Ethics Committee of the Medical College of Zhengzhou University and informed consent was obtained from the patients prior to sample collection, conforming to the Declaration of Helsinki and the local legislation. Written informed consent was obtained from all patients prior to the start of the study.

Cell culture. Human gastric cancer MKN-45, SGC-7901 and MGC-803 cell lines were obtained from the American Type Culture Collection (Manassas, VA, USA), while the human gastric epithelial mucosa GES-1 cell line was purchased from the Shanghai Institutes for Biological Sciences of the Chinese Academy of Sciences (Shanghai, China). The cells were cultured in RPMI-1640 medium (BioTeke Corporation, Beijing, China) supplemented with $10 \%$ heat-inactivated Invitrogen fetal bovine serum (FBS; Thermo Fisher Scientific, Inc., Waltham, MA, USA), $100 \mathrm{U} / \mathrm{ml}$ penicillin and $100 \mu \mathrm{g} / \mathrm{ml}$ streptomycin (Sigma-Aldrich, St. Louis, MO, USA) in a humidified cell incubator with $5 \% \mathrm{CO}_{2}$ at $37^{\circ} \mathrm{C}$.

Plasmids and cell transfection. A miR-524-5p mimic and inhibitor, alongside their corresponding negative controls (scramble miRNA), were purchased from Shanghai GenePharma Co., Ltd. (Shanghai, China). The SGC-7901 and MGC-803 cells were seeded in 6-well plates at $30 \%$ confluence one day prior to transfection. The cells were transfected with miR-524-5p mimic, miR-524-5p inhibitor and control miRNA using Invitrogen Lipofectamine ${ }^{\circledR} 2000$ (Thermo Fisher Scientific, Inc.), according to the manufacturer's protocol.

Reverse transcription-quantitative polymerase chain reaction $(R T-q P C R)$. Total RNA was extracted from the cell lines and the tissue samples using Invitrogen TRIzol reagent (Thermo Fisher Scientific, Inc.) and DNAse (catalog no. ab32124; Abcam, Cambridge, MA, USA). RT was performed using the PrimeScript $^{\mathrm{TM}}$ RT reagent Kit with gDNA Eraser (Takara Biotechnology Co., Ltd., Dalian, China), according to the manufacturer's protocol. The expression of miR-524-5p was verified by stem-loop RT-qPCR with specific RT and PCR primers. The primer sequences (Sangon, Shanghai, China) were: Matrix metallopeptidase (MMP)-2, sense, 5'-CCCCAG ACAGGTGATCTTGAC-3' and antisense, 5'-GCTTGCGAG GGAAGAAGTTG-3'; and MMP-9, sense, 5'-CGCTGGGCT TAGATCATTCC-3' and antisense, 5'-AGGTTGGATACA
TCACTGCATTAGG-3'. U6 small nuclear RNA was used as an internal control. RT-qPCR for MMP-2 and MMP-9 was performed using the following conditions: $95^{\circ} \mathrm{C}$ for $2 \mathrm{~min}$, followed by 40 cycles of $95^{\circ} \mathrm{C}$ for $15 \mathrm{sec}$ and $60^{\circ} \mathrm{C}$ for $30 \mathrm{sec}$. qPCR was performed on an Applied Biosystems ${ }^{\circledR} 7500$ thermocycler (Thermo Fisher Scientific, Inc.) using SYBR ${ }^{\circledR}$ Premix Ex Taq $^{\mathrm{TM}}$ (Tli RNaseH Plus) (Takara Biotechnology Co., Ltd.), according to the manufacturer's protocol. The comparative CT method, $\Delta \Delta \mathrm{Ct}$, was used to quantify the data. Briefly, $\Delta \mathrm{Ct}$ was calculated by subtracting the CT of U6 or GAPDH mRNA from the mRNA of interest, and $\Delta \Delta \mathrm{Ct}$ was calculated by subtracting the $\Delta \mathrm{Ct}$ of the negative control from the $\Delta \mathrm{Ct}$ of the samples. The data was normalized according to the study conducted by Schmittgen and Livak (11).

Western blot analysis. Transfected cells were washed with ice-cold phosphate-buffered saline (PBS) and lysed using a cell lysis buffer consisting of $50 \mathrm{mM}$ Tris (pH 8.0), $120 \mathrm{mM}$ $\mathrm{NaCl}, 0.5 \% \mathrm{NP}-40,50 \mathrm{mM} \mathrm{NaF}$ and $1 \mathrm{mM}$ phenylmethylsulfonyl fluoride (Beyotime Institute of Biotechnology, Haimen, China). The cells were centrifuged at $10,000 \mathrm{xg}$ for $15 \mathrm{~min}$ at $4^{\circ} \mathrm{C}$. The protein concentration was measured by BCA Protein Assay Kit (Beyotime Institute of Biotechnology). Cell protein lysates were separated on a $12 \%$ sodium dodecyl sulfate-polyacrylamide gel with the Mini-PROTEAN ${ }^{\circledR}$ II Electrophoresis System (Bio-Rad Laboratories, Inc., Hercules, CA, USA) using Mini-Protean precast gels (Bio-Rad Laboratories, Inc.) at $200 \mathrm{~V}$ for $1 \mathrm{~h}$, and subsequently transferred onto a polyvinylidene difluoride membrane (EMD Millipore, Billerica, MA, USA). The membranes were next blocked with 5\% skimmed milk in Tris-buffered saline ( $\mathrm{pH}$ 7.4) containing $0.05 \%$ Tween 20 (TBST), and incubated with primary antibodies, including anti-MMP-2 (mouse; monoclonal; dilution, 1:10; catalog no. ab80737; Abcam, Cambridge, UK), anti-MMP-9 (mouse; monoclonal; dilution, 1:25; catalog no. ab51203; Abcam) and anti- $\beta$-actin (mouse; monoclonal; dilution, 1:5000; catalog no. ab75373; Abcam) overnight at $4^{\circ} \mathrm{C}$. The membranes were washed three times in TBST for $10 \mathrm{~min}$, and subsequently incubated with HRP conjugated goat anti-rabbit IgG secondary antibodies (catalog no. MBS560261; Mybiosource, San Diego, CA, USA). Chemiluminescence detection was performed using Pierce ${ }^{\mathrm{TM}}$ Fast Western Blot kit (Thermo Fisher Scientific, Inc.) and ECL Substrate (GE Healthcare Life Sciences, Chalfont, UK). The densitometry analysis was analyzed using Image-Pro Plus software, version 1.61.

Cell proliferation assays. The transfected cells were seeded onto 96-well plates (Corning Incorporated, Corning, NY, USA) at a density of $1 \times 10^{4}$ cells/well. In total, $20 \mu 13$-(4,5-dimethylthiazol-2-yl)-2,5-diphenyltetrazolium (MTT; Sigma-Aldrich) at a concentration of $5 \mathrm{mg} / \mathrm{ml}$ was added to the transfected cells, and incubated at $37^{\circ} \mathrm{C}$ for $4 \mathrm{~h}$. Following the removal of the culture medium, the remaining crystals were dissolved in dimethyl sulfoxide (Sigma-Aldrich), and the absorbance at $570 \mathrm{~nm}$ was measured. All experiments were performed in triplicate.

Cell apoptosis. Cell apoptosis analyses were performed as previously described, with certain modifications (12). Briefly, SGC-7901 and MGC-803 cells were detached using trypsin 
A

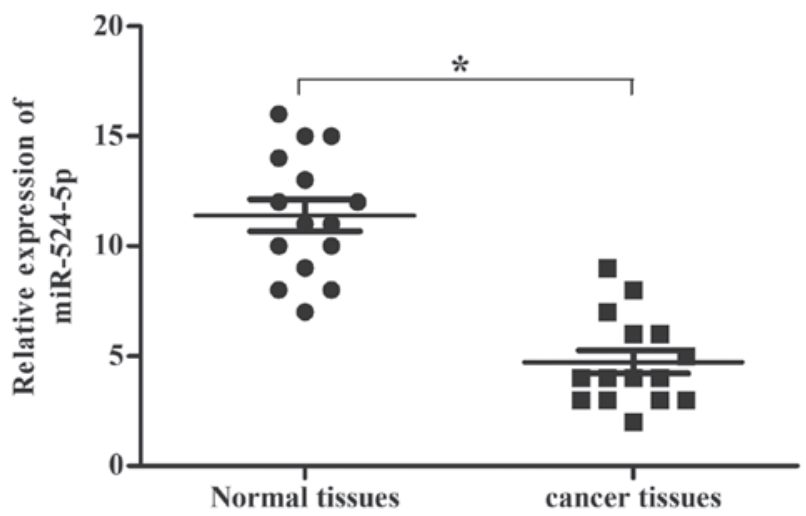

B

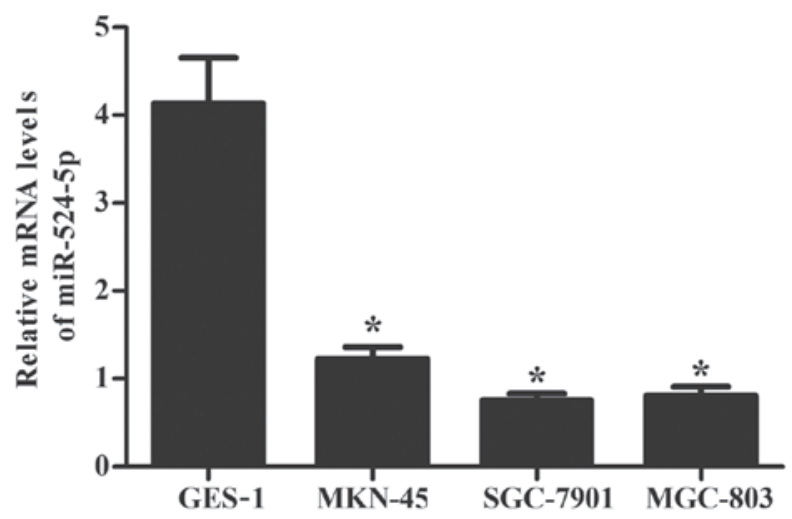

Figure 1. Expression of miR-524-5p in human gastric cancer tissues and cell lines. (A) miR-524-5p expression in 15 pairs of gastric cancer and adjacent non-cancerous tissues was assessed by RT-qPCR. *P<0.05 vs. normal tissues. (B) miR-524-5p expression in human gastric cancer MKN-45, SGC-7901 and MGC-803 cell lines, and human normal gastric epithelial mucosa GES-1 cell line was evaluated using RT-qPCR. *P<0.05 vs. GES-1 cells. All data are reported as the mean \pm standard deviation of 3 independent experiments. miR, microRNA; mRNA, messenger RNA; RT-qPCR, reverse transcription-quantitative polymerase chain reaction.

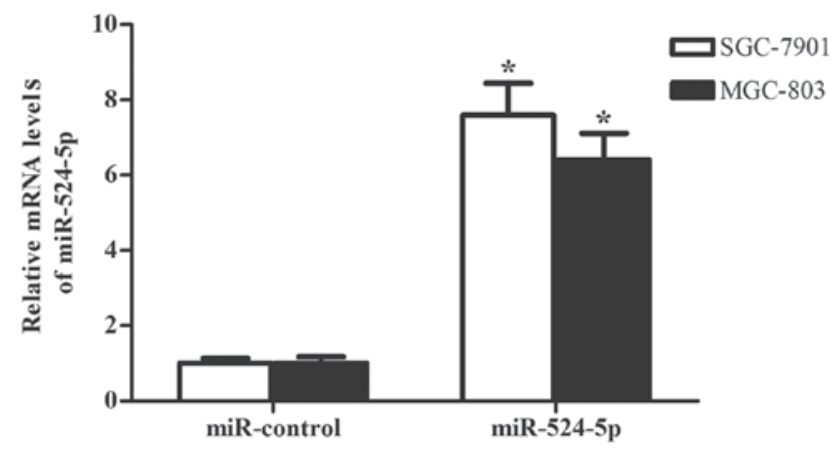

Figure 2. Determination of transfection efficiency. miR-524-5p expression was detected in human gastric cancer SGC-7901 and MGC-803 cells using reverse transcription-quantitative polymerase chain reaction $48 \mathrm{~h}$ following transfection with miR-control and miR-524-5p mimic. All experiments were repeated $\geq 3$ times and all data are reported as the mean \pm standard deviation. "P $<0.05$ vs. miR-control. miR, microRNA; mRNA, messenger RNA.

(Sigma-Aldrich), washed twice in PBS, centrifuged at $1,000 \mathrm{xg}$ for $5 \mathrm{~min}$ and resuspended in $195 \mu \mathrm{l}$ Annexin V-fluorescein isothiocyanate (FITC) binding buffer (Sigma-Aldrich). Subsequently, $5 \mu \mathrm{l}$ Annexin V-FITC (BD Biosciences, Erembodegem, Belgium) was added to the cells. Following an incubation period of $15 \mathrm{~min}$ in the dark at room temperature, $400 \mu \mathrm{l}$ binding buffer was added. The percentage of apoptotic cells was analyzed using flow cytometry (FACSCalibur ${ }^{\mathrm{TM}}$; BD Biosciences, Franklin Lakes, NJ, USA) and CellQuest Pro ${ }^{\mathrm{TM}}$ Software (version 5.2.1; BD Biosciences).

Cell invasion assays. Cell invasion was determined using 24-well Transwell chambers coated with Matrigel ${ }^{\mathrm{TM}}$ (BD Biosciences, Bedford, MD, USA) in order to determine the effect of miR-524-5p on gastric cancer cell invasion. Briefly, $8-\mu \mathrm{m}$ pore size filters (Sigma-Aldrich) were coated with $100 \mu \mathrm{l}$ Matrigel $^{\mathrm{TM}}$ (1 mg/ml; dissolved in serum-free RPMI-1640). A total of $600 \mu \mathrm{l}$ RPMI-1640 containing 10\% Invitrogen FBS (Thermo Fisher Scientific) was added to the lower chamber, while single cell suspensions $\left(1 \times 10^{5}\right.$ cells/well) were added to the upper chamber. Cells were incubated for $48 \mathrm{~h}$ at $37^{\circ} \mathrm{C}$, and non-invading cells were removed using cotton swabs (Takara
Biotechnology Co. Ltd.). Invaded cells were stained with $0.1 \%$ crystal violet (Takara Biotechnology Co., Ltd.) for $10 \mathrm{~min}$ at room temperature and examined using the Olympus BX51 fluorescence microscope (Olympus Optical Co., Tokyo, Japan).

Statistical analysis. The results are presented as the mean \pm standard deviation. The Student's $t$-test was used to assess the statistical significance of differences between groups. The data were analyzed by SPSS version 11.0 software (SPSS, Inc., Chicago, IL, USA). $\mathrm{P}<0.05$ was considered to indicate a statistically significant difference.

\section{Results}

Expression of miR-524-5p in gastric cancer tissues and cell lines. In order to determine the expression levels of miR-524-5p in gastric cancer, the present study evaluated the expression levels of miR-524-5p in human gastric cancer tissues and cell lines using RT-qPCR. As demonstrated by Fig. 1A, the expression levels of miR-524-5p were markedly lower in gastric cancer tissues, compared with normal gastric mucosa tissue. Similarly, the expression levels of miR-524-5p were significantly decreased in MKN-45, SGC-7901 and MGC-803 cells, compared with human normal gastric epithelial mucosa GES-1 cells (Fig. 1B). These results demonstrate that $\mathrm{miR}-524-5 \mathrm{p}$ is downregulated in gastric cancer tissues and cell lines. SGC-7901 and MGC-803 cells were selected for the subsequent in vitro experiments, since the mRNA expression levels of miR-524-5p were lower in these cells than in MKN-45 cells.

Effects of miR-524-5p on gastric cancer cell proliferation. Since miR-524-5p was significantly downregulated in gastric cancer, the present study concluded that miR-524-5p may inhibit growth, promote apoptosis and enhance invasion in gastric cancer cells. To verify this hypothesis, the expression levels of miR-524-5p in SGC-7901 and MGC-803 cells were upregulated by transfecting the cells with miR-524-5p mimics, as detected by RT-qPCR (Fig. 2). Subsequently, an MTT assay was performed to evaluate the effects of miR-524-5p on the 

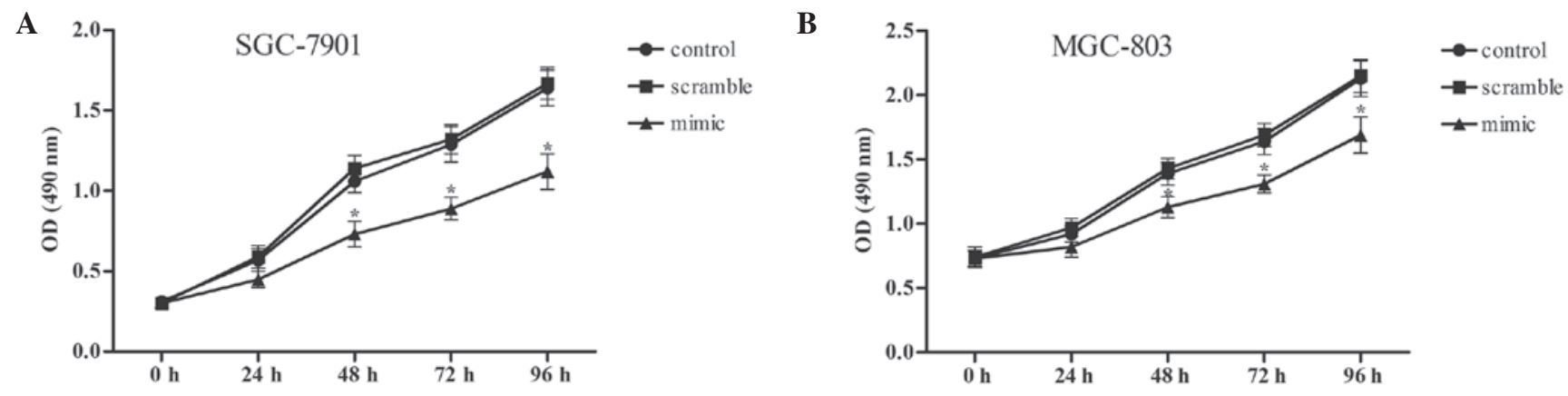

Figure 3. Effects of miR-524-5p on gastric cancer cell proliferation. To evaluate the effects of miR-524-5p on the growth of human gastric cancer SGC-7901 and MGC-803 cells, a 3-(4,5-dimethylthiazol-2-yl)-2,5-diphenyltetrazolium assay was conducted every $24 \mathrm{~h}$ from 0 to $96 \mathrm{~h}$ following transfection. miR-524-5p overexpression inhibited the proliferation of (A) SGC-7901 and (B) MGC-803 cells, compared with control and scramble miRNA groups. All experiments were repeated $\geq 3$ times and all data are reported as the mean \pm standard deviation. " $\mathrm{P}<0.05$ vs. miR-control. miR, microRNA; mRNA, messenger RNA; OD, optical density.

A

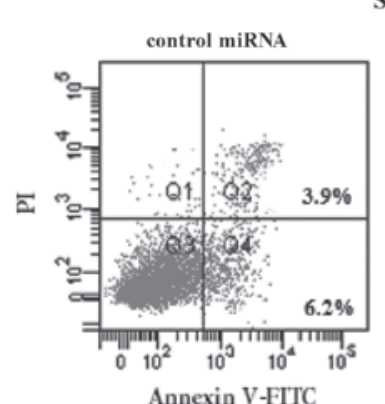

SGC-7901

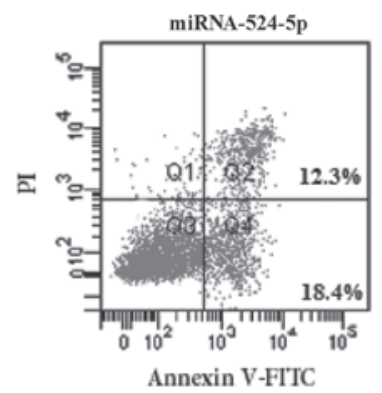

$\mathbf{B}$

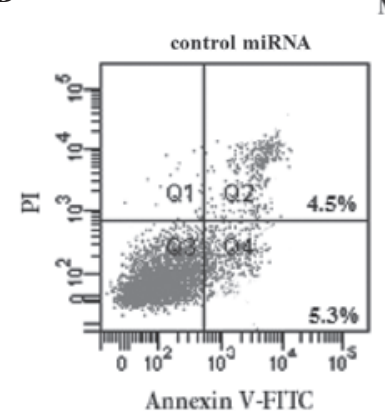

MGC-803

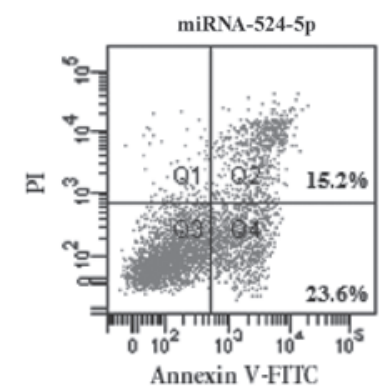

C

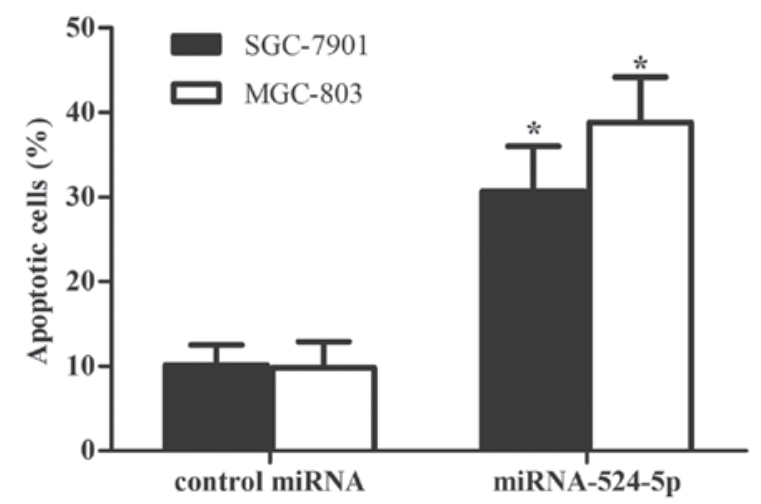

Figure 4. Effect of miR-524-5p on gastric cancer cell apoptosis. The percentage of apoptotic cells in human gastric cancer (A) SGC-7901 and (B) MGC-803 cells was detected by flow cytometry using Annexin V-FITC and PI staining following $48 \mathrm{~h}$ of miR-524-5p mimic transfection. (C) Data are reported as the mean \pm standard deviation of 3 independent experiments. ${ }^{*} \mathrm{P}<0.05$ vs. miR-control. miR, microRNA; miRNA, microRNA; FITC, fluorescein isothiocyanate; PI, propidium iodide. growth of SGC-7901 and MGC-803 cells. As demonstrated by Fig. 3, miR-524-5p overexpression inhibited the growth of SGC-7901 and MGC-803 cells, and the inhibitory effect of miR-524-5p was markedly increased with increasing transfection times.

Effect of miR-524-5p on gastric cancer cell apoptosis. Cell apoptosis was detected using propidium iodide and Annexin V-FITC staining following $48 \mathrm{~h}$ of miR-524-5p mimic transfection. The percentage of cells undergoing apoptosis was measured using flow cytometry. Following transfection with miR-524-5p mimics, the percentage of apoptosis significantly increased to $30.7 \%$ in SGC-7901 cells (Fig. 4A) and 38.8\% in MGC-803 cells (Fig. 4B), compared with 10.1 and $9.8 \%$, respectively, in the control groups. These results demonstrate that miR-524-5p is critical in promoting the apoptosis of gastric cancer cells.

Effects of miR-524-5p on gastric cancer cell invasion. The present study examined the effect of miR-524-5p on gastric cancer cell invasion. As demonstrated by Fig. 5, the number of invasive cells was significantly decreased in SGC-7901 and MGC-803 cells transfected with miR-524-5p mimics, compared with miR-control-transfected cells (SGC-7901 miR-524-5p-transfected vs. control cells, 85 vs. 173 cells; MGC-803 miR-524-5p-transfected vs. control cells, 139 vs. 222 cells). These results indicate that overexpression of miR-524-5p inhibits the invasion ability of gastric cancer cells in vitro.

miR-524-5p transfection attenuates cell invasion by inhibiting the expression of MMP-2 and MMP-9. Numerous studies have demonstrated that extracellular MMP-2 and MMP-9 are overexpressed in various types of cancer (13-15). As demonstrated in Fig. 6, the protein expression levels of MMP-2 and MMP-9 were significantly decreased in SGC-7901 (Fig. 6A) and MGC-803 (Fig. 6B) cells transfected with miR-524-5p mimics. Similarly to the results of western blot analysis, a decrease in the mRNA levels of MMP-2 and MMP-9 was observed using RT-qPCR (Fig. 6C and D). These results demonstrate that transfection with miR-524-5p attenuates gastric cancer cell invasion by inhibiting the expression of MMP-2 and MMP-9. 
A

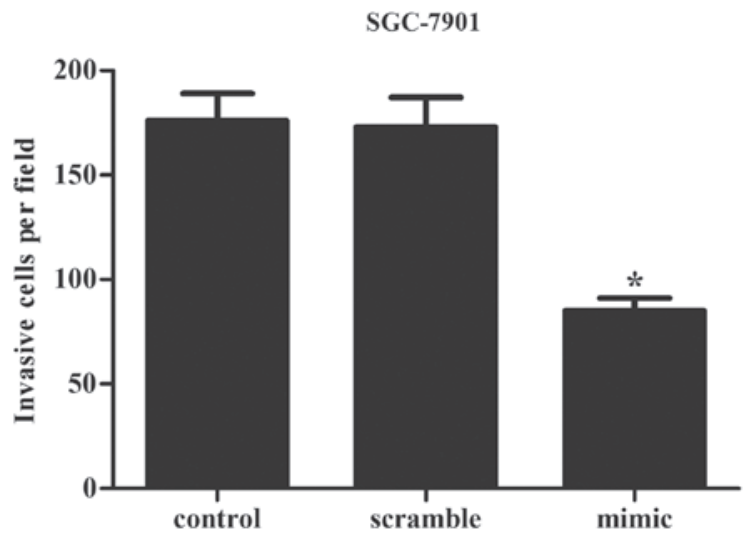

B

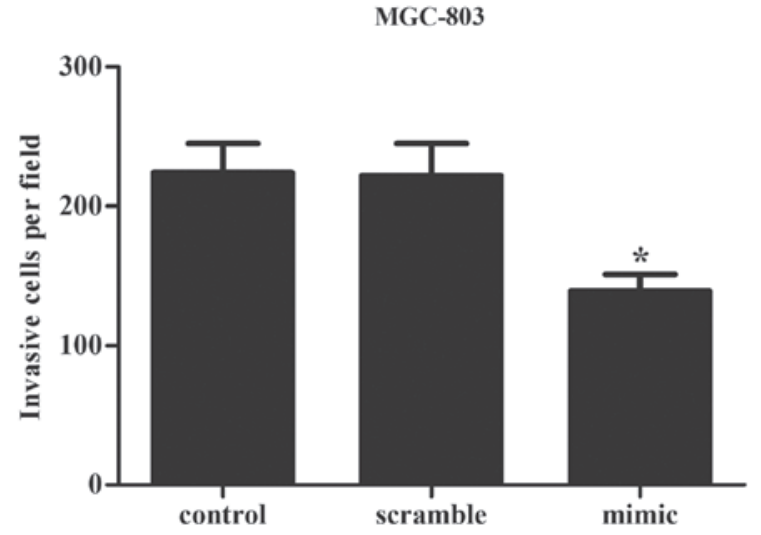

Figure 5. Effects of miR-524-5p on gastric cancer cell invasion. Cell invasion was measured using a Transwell assay 48 h following miR-524-5p transfection. miR-524-5p overexpression inhibited the invasion of human gastric cancer (A) SGC-7901 and (B) MGC-803 cells, compared with control cells. All experiments were repeated $\geq 3$ times and the data are reported as the mean \pm standard deviation. ${ }^{*} \mathrm{P}<0.05$ vs. miR-control. miR, microRNA.

A

SGC-7901

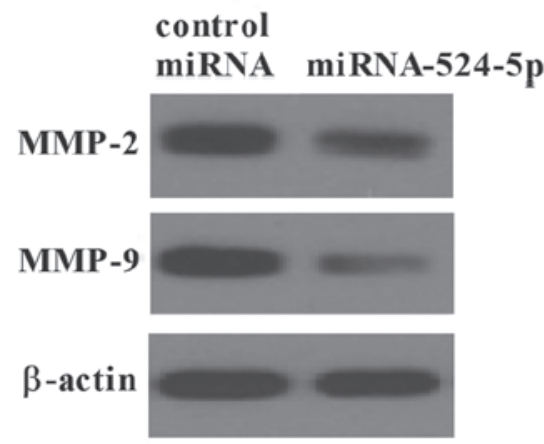

C

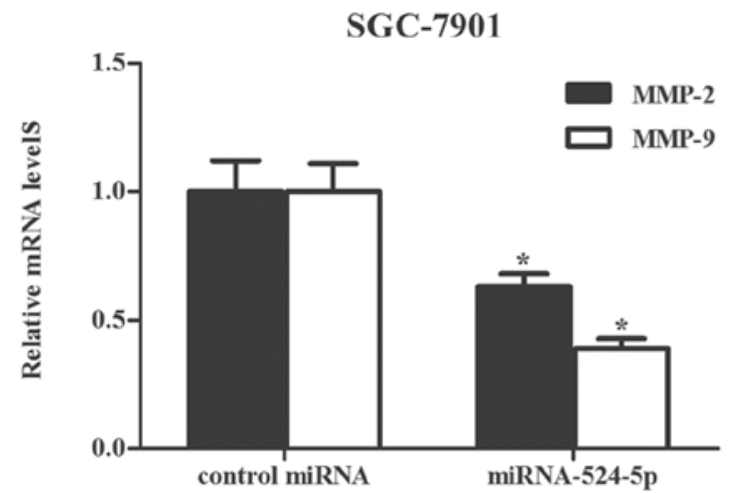

B

MGC-803

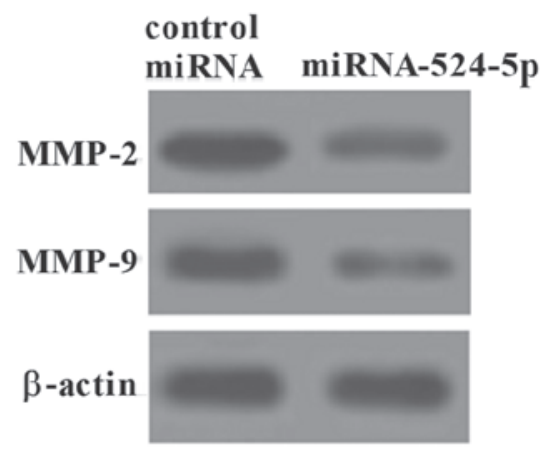

D

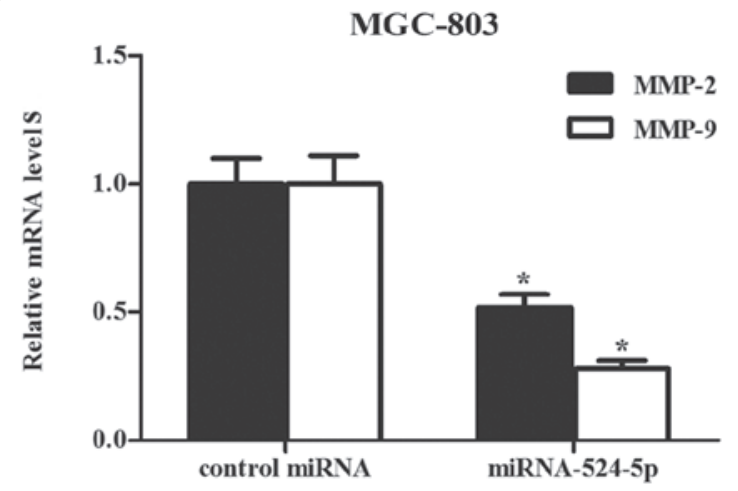

Figure 6. miR-524-5p transfection attenuates cell invasion by inhibiting the expression of MMP-2 and MMP-9. miR-524-5p overexpression decreased the protein levels of MMP-2 and MMP-9 in human gastric cancer (A) SGC-7901 and (B) MGC-803 cells. miR-524-5p overexpression decreased the messenger RNA levels of MMP-2 and MMP-9 in (C) SGC-7901 and (D) MGC-803 cells. All experiments were repeated $\geq 3$ times and the data are reported as the mean \pm standard deviation. ${ }^{*}<0.05$ vs. miR-control; miR, microRNA; miRNA, microRNA; mRNA, messenger RNA; MMP, matrix metallopeptidase.

\section{Discussion}

Previous studies have demonstrated that the aberrant expression of miRNAs is associated with the development of various types of human cancer. These studies have also indicated that miRNAs may function as oncogenes or tumor-suppressor genes $(16,17)$. In addition, previous studies have identified numerous dysregulated miRNAs, including miR-30b,
miR-372, miR-126 and miR-21, that modulate growth, apoptosis, migration and invasion in gastric cancer cells (18-23). The present study revealed that the expression levels of miR-524-5p were downregulated in gastric cancer tissues and cell lines, and overexpression of miR-524-5p inhibited gastric cancer cell proliferation and invasion and promoted cell apoptosis. Therefore, the present study suggests that miR-524-5p functions as a tumor suppressor in human gastric cancer. 
Dysregulation of miR-524-5p is a frequent event in several types of cancer, including a reduction in the expression of miR-524-5p in melanoma (10) and glioma cells (9). The present study demonstrated that the expression levels of miR-524-5p were downregulated in gastric cancer tissues and cell lines, suggesting that miR-524-5p functions as a tumor suppressor in human gastric cancer, which is consistent with previous studies (24).

Apoptosis is programmed cell-death that occurs in physiological and pathological conditions (25). A disruption in cell apoptosis may result in the development of cancer (26). The current study revealed that ectopic expression of miR-524-5p induced a greater percentage of gastric cancer cells to undergo apoptosis, compared with control cells in vitro. These results suggest that miR-524-5p may be involved in gastric cancer by promoting the apoptosis of cancer cells.

In patients with gastric cancer, metastasis is one of the leading causes of mortality (27). Since miR-524-5p was observed to be downregulated in gastric cancer tissues and cell lines, the present study hypothesized that overexpressing miR-524-5p may suppress the metastasis of gastric cancer cells. The results of the present study demonstrated that overexpression of miR-524-5p in human gastric cancer SGC-7901 and MGC-803 cells significantly inhibited metastasis. These findings suggest an inhibitory role for miR-524-5p in the development of metastasis in gastric cancer, which is, to the best of our knowledge, a novel result. In addition, the results of the present study indicate that decreased expression of miR-524-5p in gastric cells may contribute to the development of gastric cancer.

Degradation of the extracellular matrix (ECM) in blood or lymph vessels is critical to develop metastasis, since the loss of the ECM allows the cancer cells to invade the blood or lymphatic system and spread to distant tissues and organs (28). MMPs are a family of zinc-dependent enzymes that are essential for the progression of cancer (29). MMP-2 is expressed on the surface of tumor cells, and is involved in tumor metastasis by activating pro-MMP-2, which exacerbates the malignancy (30). The activation of pro-MMP-2 by membrane-type 1 matrix metalloproteinase (MT1-MMP) is considered to be a critical event in cancer cell invasion. In the activation step, TIMP metallopeptidase inhibitor 2 bound to MT1-MMP on the cell surface acts as a receptor for pro-MMP-2. MT1-MMP is expressed on the cancer cell surface as an invasion-promoting proteinase. By localizing at the leading edge of invasive cancer cells, MT1-MMP degrades components of the tissue barriers. One of the major targets is type I collagen, the most abundant ECM component. Although MT1-MMP itself cannot degrade type IV collagen in the basement membrane, it binds to and activates proMMP-2, one of the type IV collagenases. However, degradation of the ECM is not the sole function of MT1-MMP (31). MMP-2 is considered an important marker for the presence of distant metastasis in cancer (32). MMP-9 is also responsible for the degradation of the ECM, and is pivotal in tumor invasion and metastasis (33). The activated MMP-9 enhances the invasive phenotype of the cultured cells as their ability to both degrade extracellular matrix and transverse basement membrane is significantly increased following zymogen activation.
Liao et al (34) reported that miRNA-196 (miR-196) is highly expressed in gastric cancer, and overexpression of miR-196 significantly induced migration and invasion of gastric cancer cells by decreasing the levels of MMP-2 and MMP-9. By contrast, the present study investigated whether the inhibitory role of miR-524-5p on cell invasion occurs through regulating the expression of MMP-2 and MMP-9. The present study revealed that miR-524-5p downregulated the expression levels of MMP-2 and MMP-9, which explains the inhibition of invasion caused by miR-524-5p in gastric cells during the invasion assay.

In summary, the results of the present study indicate that miR-524-5p is a tumor suppressor gene that is involved in the development of gastric cancer. Overexpression of miR-524-5p inhibits cell proliferation and invasion, and promotes apoptosis in gastric cancer cells. Therefore, miR-524-5p may be a novel therapeutic target for the treatment of gastric cancer.

\section{References}

1. Ferlay J, Soerjomataram I, Dikshit R, Eser S, Mathers C, Rebelo M, Parkin DM, Forman D and Bray F: Cancer incidence and mortality worldwide: Sources, methods and major patterns in GLOBOCAN 2012. Int J Cancer 136: E359-E386, 2015.

2. Ferlay J, Shin HR, Bray F, Forman D, Mathers C and Parkin DM: Estimates of worldwide burden of cancer in 2008: GLOBOCAN 2008. Int J Cancer 127: 2893-2917, 2010.

3. Siegel R, Ma J, Zou Z and Jemal A: Cancer statistics, 2014. CA Cancer J Clin 64: 9-29, 2014.

4. Yan B, Yau EX, Bte Omar SS, Ong CW, Pang B, Yeoh KG and Salto-Tellez M: A study of HER2 gene amplification and protein expression in gastric cancer. J Clin Pathol 63: 839-842, 2010.

5. Yamanoi K, Fukuma M, Uchida H, Kushima R, Yamazaki K, Katai H, Kanai Y and Sakamoto M: Overexpression of leucine-rich repeat-containing $\mathrm{G}$ protein-coupled receptor 5 in gastric cancer. Pathol Int 63: 13-19, 2013.

6. Ning X, Sun S, Zhang K, Liang J, Chuai Y, Li Y and Wang X: S100A6 protein negatively regulates CacyBP/SIP-mediated inhibition of gastric cancer cell proliferation and tumorigenesis. PLoS One 7: e30185, 2012.

7. Baek D, Villén J, Shin C, Camargo FD, Gygi SP and Bartel DP: The impact of microRNAs on protein output. Nature 455: 64-71, 2008.

8. Bartel DP: MicroRNAs: Genomics, biogenesis, mechanism, and function. Cell 116: 281-297, 2004.

9. Chen L, Zhang W, Yan W, Han L, Zhang K, Shi Z, Zhang J, Wang Y, Li Y, Yu S, et al: The putative tumor suppressor miR-524-5p directly targets Jagged-1 and Hes-1 in glioma. Carcinogenesis 33: 2276-2282, 2012.

10. Liu SM, Lu J, Lee HC, Chung FH and Ma N: miR-524-5p suppresses the growth of oncogenic BRAF melanoma by targeting BRAF and ERK2. Oncotarget 5: 9444-9459, 2014.

11. Schmittgen T D and Livak K J: Analyzing real-time PCR data by the comparative CT method. Nat Protoc 3: 1101-1108, 2008.

12. Hou T, Ou J, Zhao X, Huang X, Huang Y and Zhang Y: MicroRNA-196a promotes cervical cancer proliferation through the regulation of FOXO1 and p27Kip1. Br J Cancer 110: 1260-1268, 2014

13. Gao J, Ding F, Liu Q and Yao Y: Knockdown of MACC1 expression suppressed hepatocellular carcinoma cell migration and invasion and inhibited expression of MMP2 and MMP9. Mol Cell Biochem 376: 21-32, 2013.

14. Liu Q, Yang P, Tu K, Zhang H, Zheng X, Yao Y and Liu Q: TPX2 knockdown suppressed hepatocellular carcinoma cell invasion via inactivating AKT signaling and inhibiting MMP2 and MMP9 expression. Chin J Cancer Res 26: 410-417, 2014.

15. Huang Q, Lan F, Wang X, Yu Y, Ouyang X, Zheng F, Han J, Lin Y, Xie Y, Xie F, et al: IL-1 $\beta$-induced activation of p38 promotes metastasis in gastric adenocarcinoma via upregulation of AP-1/c-fos, MMP2 and MMP9. Mol Cancer 13: 18, 2014.

16. Lin S and Gregory RI: MicroRNA biogenesis pathways in cancer. Nat Rev Cancer 15: 321-333, 2015. 
17. Yao Y, Suo AL, Li ZF, Liu LY, Tian T, Ni L, Zhang WG, Nan KJ, Song TS and Huang C: MicroRNA profiling of human gastric cancer. Mol Med Rep 2: 963-970, 2009.

18. Croce CM: Causes and consequences of microRNA dysregulation in cancer. Nat Rev Genet 10: 704-714, 2009.

19. Hwang HW and Mendell JT: MicroRNAs in cell proliferation, cell death, and tumorigenesis. Br J Cancer 94: 776-780, 2006.

20. Zhu ED, Li N, Li BS, Li W, Zhang WJ, Mao XH, Guo G, Zou QM and Xiao B: miR-30b, down-regulated in gastric cancer, promotes apoptosis and suppresses tumor growth by targeting plasminogen activator inhibitor-1. PLoS One 9: e106049, 2014.

21. Cho WJ, Shin JM, Kim JS, Lee MR, Hong KS, Lee JH, Koo KH, Park JW and Kim KS: miR-372 regulates cell cycle and apoptosis of ags human gastric cancer cell line through direct regulation of LATS2. Mol Cells 28: 521-527, 2009.

22. Feng R, Chen X, Yu Y, Su L, Yu B, Li J, Cai Q, Yan M, Liu B and Zhu Z: miR-126 functions as a tumour suppressor in human gastric cancer. Cancer Lett 298: 50-63, 2010.

23. Zhang Z, Li Z, Gao C, Chen P, Chen J, Liu W, Xiao S and Lu H: miR-21 plays a pivotal role in gastric cancer pathogenesis and progression. Lab Invest 88: 1358-1366, 2008.

24. Liu SM, Lu J, Lee HC, Chung FH and Ma N: miR-524-5p suppresses the growth of oncogenic BRAF melanoma by targeting BRAF and ERK2. Oncotarget 5: 9444-9459, 2014.

25. Elmore S: Apoptosis: A review of programmed cell death. Toxicol Pathol 35: 495-516, 2007.

26. Cotter TG: Apoptosis and cancer: The genesis of a research field. Nat Rev Cancer 9: 501-507, 2009.
27. Bang YJ, Kim YW, Yang HK, Chung HC, Park YK, Lee KH, Lee KW, Kim YH, Noh SI, Cho JY, et al; CLASSIC trial investigators: Adjuvant capecitabine and oxaliplatin for gastric cancer after D2 gastrectomy (CLASSIC): A phase 3 open-label, randomised controlled trial. Lancet 379: 315-321, 2012.

28. Chen K, Zhang S, Ji Y, Li J, An P, Ren H, Liang R, Yang J and Li Z: Baicalein inhibits the invasion and metastatic capabilities of hepatocellular carcinoma cells via down-regulation of the ERK pathway. PLoS One 8: e72927, 2013.

29. Lee SJ, Kim WJ and Moon SK: Role of the p38 MAPK signaling pathway in mediating interleukin-28A-induced migration of UMUC-3 cells. Int J Mol Med 30: 945-952, 2012

30. Egeblad M and Werb Z: New functions for the matrix metalloproteinases in cancer progression. Nat Rev Cancer 2: 161-174, 2002.

31. Seiki M: Membrane-type 1 matrix metalloproteinase: A key enzyme for tumor invasion. Cancer Lett 194: 1-11, 2003.

32. Ueda J, Kajita M, Suenaga N, Fujii K and Seiki $M$ : Sequence-specific silencing of MT1-MMP expression suppresses tumor cell migration and invasion: Importance of MT1-MMP as a therapeutic target for invasive tumors. Oncogene 22: 8716-8722, 2003.

33. Deryugina EI and Quigley JP: Matrix metalloproteinases and tumor metastasis. Cancer Metastasis Rev 25: 9-34, 2006.

34. Liao YL, Hu LY, Tsai KW, Wu CW, Chan WC, Li SC, Lai CH, Ho MR, Fang WL, Huang KH and Lin WC: Transcriptional regulation of miR-196b by ETS2 in gastric cancer cells. Carcinogenesis 33: 760-769, 2012. 